\title{
Propagation of States in Dilation Analytic Potentials and Asymptotic Completeness
}

\author{
Peter A. Perry * \\ Department of Physics, Princeton University, Princeton, NJ 08544, USA
}

\begin{abstract}
We estimate the space-time behavior of scattering states for two-body Schrödinger operators with smooth, dilation analytic potentials. We use our estimates to give a simple proof of asymptotic completeness for a class of longrange potentials, including the Coulomb potential plus a fairly general shortrange perturbation.
\end{abstract}

\section{Introduction}

The goal of this paper is to present a simple proof of asymptotic completeness for the modified wave operators that describe two-body quantum scattering with certain long-range potentials. Modified wave operators were introduced by Dollard [6] to study scattering for the Coulomb potential. Spectral and scattering theory for general long-range potentials has since been studied by many authors. Spectral representations for such long-range Schrödinger operators have been studied by Ikebe $[14,15]$ and Saito $[31,32]$. Their results imply completeness of the stationary wave operators defined via the spectral representation. Isozaki [18] proved completeness of the stationary wave operator and Kitada [22-24] proved completeness of time-dependent modified wave operators by a stationary method. More recently Ikebe and Isozaki $[16,17]$ have also given a proof of completeness for the modified wave operators. Agmon [1] has also proved completeness results for Schrödinger operators with long-range potentials and Enss [8] has given a "geometric" proof of completeness for certain long-range potentials.

Here we would like to give a simple, "geometric" proof of completeness for Schrödinger operators $H_{1}=H_{0}+V+\bar{V}$ on $L^{2}\left(\mathbb{R}^{n}\right)$, where $H_{0}=-\frac{1}{2} \Delta, V$ is a longrange, dilation analytic potential, and $\bar{V}$ is a fairly general short range perturbation (we formulate precise hypotheses below). Our class of potentials thus includes the Coulomb potential plus a fairly general short-range perturbation. Our assumptions

* Present address: Courant Institute of Mathematical Sciences, 251 Mercer Street, New York, NY 10012, USA

Supported by USNSF Grant MCS-78-01885 
are more restrictive than those of the authors mentioned above in that we require the long-range potential to be dilation analytic, but on the other hand we can allow a more general short range part.

The modified wave operators we will study are given by:

$$
\Omega_{\bar{D}}^{ \pm}\left(H_{1}, H_{0}\right)=\mathrm{s}-\lim _{t \rightarrow \mp \infty} \mathrm{e}^{i H_{1} t} \mathscr{U}_{0}(t, 0),
$$

where the "modified free evolution" $\mathscr{U}_{0}(t, s)$ is defined by

$$
\mathscr{U}_{0}(t, s)=\exp -i\left\{H_{0}(t-s)+\int_{s}^{t} W^{\prime}(\mathbf{p} \tau) \mathrm{d} \tau\right\}
$$

In (2) $W^{\prime}$ is a smooth function that closely approximates the long-range behavior of $V$ (we choose $W^{\prime}$ in Proposition 1.2 below). $\mathbf{p}$ is the momentum operator and, with our choice of $H_{0}, \mathbf{p}=\mathbf{v}$, the velocity operator.

The class of dilation analytic potentials was introduced in [2]; see [4] for a characterization of dilation analytic potentials and [30] for discussion and further references. We denote by $\mathscr{U}(\theta)$ the group of dilations: $\mathscr{U}(\theta)$ acts on $L^{2}\left(\mathbb{R}^{n}\right)$ by $(\mathscr{U}(\theta) \psi)(x)=\mathrm{e}^{n \theta / 2} \psi\left(\mathrm{e}^{\theta} x\right)$ for vectors $\psi \in L^{2}\left(\mathbb{R}^{n}\right)$. A symmetric, $H_{0}$-compact operator is a dilation analytic potential if the operator $\mathscr{U}(\theta) V \mathscr{U}(\theta)^{-1}\left(H_{0}+i\right)^{-1}$ extends to a bounded operator-valued analytic function of $\theta$ in some strip $S_{\varphi}$ $=\{\theta:|\operatorname{Im} \theta|<\varphi\}$. We will assume that $0<\varphi<\pi / 4$. If $H=H_{0}+V$ and $V$ is dilation analytic, $H(\theta)=\mathscr{U}(\theta) H \mathscr{U}(\theta)^{-1}$ extends to an analytic family of type (A) in $S_{\varphi}$. In [2] this analyticity is used to prove, among other results, that $H$ has no singular spectrum and that eigenvalues of $H$ can accumulate only at 0 .

We are now ready to state our result.

Theorem 1. Let $H_{0}=-\frac{1}{2} \Delta$ and $H_{1}=H_{0}+V+\bar{V}$ on $L^{2}\left(\mathbb{R}^{n}\right)$. Suppose that:

(i) $V$ is dilation analytic in some strip $S_{\varphi}$ and $(1+|x|)^{1+\alpha}(\nabla V)(x)$ (distributional derivative) is uniformly locally $L^{2}$ for some $\alpha>\frac{1}{2}$.

(ii) $\left(H_{1}+i\right)^{-1}-(\tilde{H}+i)^{-1} \in \mathscr{I}_{\infty}$, the ideal of compact operators, where $\tilde{H}=H_{0}+V$.

(iii) For some integers $\beta, \gamma \geqq 1$ and some $\varepsilon>0$, the bounded, monotone decreasing function $\tilde{h}(R)=\left\|\left(H_{1}+i\right)^{-\beta} \bar{V}\left(H_{0}+i\right)^{-\gamma} F\left(|x| \geqq R^{1-\varepsilon}\right)\right\|$ is integrable on $(0, \infty)$. (Here and elsewhere, $F(x \in S)$ denotes multiplication by the characteristic function of the set $S$.)

Then the modified wave operators $\Omega_{D}^{ \pm}\left(H_{1}, H_{0}\right)$ exist and are complete, i.e., $\operatorname{Ran} \Omega_{D}^{+}=\operatorname{Ran} \Omega_{D}^{-}=\mathscr{H}_{\text {a.c. }}\left(H_{1}\right)$ and $H_{1}$ has empty singular spectrum. Eigenvalues of $H_{1}$ can accumulate only at 0 .

Remarks. 1. To treat the Coulomb potential $|x|^{-1}$, we write it as $(1+|x|)^{-1}$ $+\left[|x|^{-1}-(1+|x|)^{-1}\right]$ and group the term in square brackets with the short range potential $\bar{V}$. We can similarly treat power potentials $|x|^{-\alpha}$ for $\alpha>\frac{1}{2}$. 2. The existence theory of modified wave operators with our choice (2) of modified free evolution $[3,5]$ breaks down at $\alpha=\frac{1}{2}$, so our hypothesis (i) is necessary.

Below in Sect. 1, we will prove that the potential $V$ in Theorem 1 can be written as $V=W+\bar{W}$ where $W$ is $C^{\infty}$ and dilation analytic, $|\nabla W(x)| \leqq C(1+|x|)^{-(1+\alpha)}$, and $\bar{W}$ is short-range (Proposition 1.1). Since $W$ is smooth, the operator $H=H_{0}+W$ 
has several domain properties which are technically convenient: we collect them in Proposition 1.3.

Given Proposition 1.1, it is very natural to break up the proof of completeness of $\Omega_{D}^{ \pm}\left(H_{1}, H_{0}\right)$ into two steps: (1) prove that the ordinary wave operators $\Omega^{ \pm}\left(H_{1}, H\right)$ exist and are complete, and (2) prove that the modified wave operators $\Omega_{D}^{ \pm}\left(H, H_{0}\right)$ are complete.

The heart of our method is a "geometrical" estimate on the space-time behavior of scattering states propagating under $\exp (-i H t)$. To state it, let $D=\frac{1}{2}(\mathbf{x} \cdot \mathbf{p}+\mathbf{p} \cdot \mathbf{x})$ be the generator of dilations and let $P_{+}$(respectively $P_{-}$) project onto the positive (respectively negative) spectral subspace for $D$. Let $g$ be a smooth function with compact support in $(0, \infty)$ away from eigenvalues of $H$. In Sect. 2 we prove:

$$
\left\|F\left(|x| \leqq|t|^{1-\varepsilon}\right) \mathrm{e}^{-i t H} g(H) P_{ \pm}\right\| \leqq C_{N, \varepsilon}(1+|t|)^{-N}
$$

for any integer $N$, any $\varepsilon>0$, and $\pm t \in(0, \infty)$. Estimate (3) is proved by extending Mourre's technique in [26], where he proves a similar estimate for $H=H_{0}=-\frac{1}{2} \Delta$. "Local decay" estimates similar to our estimate (3) have been proven for certain dilation-analytic potentials in [19] and for a larger class of potentials in [20,21]. These authors do not apply their estimates to prove asymptotic completeness. Together with any formulation of Enss's method [7] for short-range scattering, (3) immediately implies:

Theorem 2. Let $H=H_{0}+W$, where:

(i)' $W$ is dilation analytic in $S_{\varphi}$ and $C^{\infty}$ with bounded derivatives. Suppose $H_{1}$ is another self-adjoint operator so that

(ii) $\left(H_{1}+i\right)^{-1}-(H+i)^{-1} \in \mathscr{I}_{\infty}$.

(iii)' $^{\prime}$ For some integers $\beta, \gamma \geqq 1$ and some $\varepsilon>0$, the bounded, monotone decreasing function $h(R)=\left\|\left(H_{1}+i\right)^{-\beta}\left(H_{1}-H\right)(H+i)^{-\gamma} F\left(|x| \geqq R^{1-\varepsilon}\right)\right\|$ is integrable on $(0, \infty)$.

Then $\Omega^{ \pm}\left(H_{1}, H\right)$ exist and are complete, i.e., $\operatorname{Ran} \Omega^{+}\left(H_{1}, H\right)=\operatorname{Ran} \Omega^{-}\left(H_{1}, H\right)$ $=\mathscr{H}_{\text {a.c. }}\left(H_{1}\right)$ and $H_{1}$ has empty singular spectrum. Eigenvalues of $H_{1}$ can accumulate only at 0 .

Remark. To show that (i)-(iii) of Theorem $1 \Rightarrow($ i)'-(iii)' of Theorem 2 when $H_{1}-H=\bar{W}+\bar{V}$, one uses Propositions 1.1 and 1.3(a). Proposition 1.3 (a) enters in showing that (iii)' holds given (iii).

The next step:

Theorem 3. Let $H=H_{0}+W$, where:

$(i)^{\prime \prime} W$ is $C^{\infty}$ with bounded derivatives and dilation analytic in $S_{\varphi}$ and $|\nabla W(x)|$ $\leqq C(1+|x|)^{-(1+\alpha)}$ for some $\alpha>\frac{1}{2}$.

Then the modified wave operators $\Omega_{D}^{ \pm}\left(H, H_{0}\right)$ are complete, i.e., $\operatorname{Ran} \Omega_{D}^{+}$ $=\operatorname{Ran} \Omega_{D}^{-}=\mathscr{H}_{\text {a.c. }}(H)$.

To prove Theorem 3, we will prove directly that the inverse modified wave operators $\Omega_{D}^{ \pm}\left(H, H_{0}\right) *$ exist as strong limits. Just as the usual "Cook's method" proof for the existence of $\Omega_{D}^{ \pm}$depends on the asymptotic equality of $\mathbf{x}$ and $\mathbf{p} t$ under the free evolution $\exp \left(-i t H_{0}\right)$, so our proof depends on the same fact with $H_{0}$ 
replaced by $H$. In Sect. 3 we combine the estimate (3) with ideas of Enss [9] to prove that $\mathbf{x}$ and $\mathbf{p} t$ are asymptotically equal under $\exp (-i t H)$. We use this result, a result of Enss on the operator $D(t)=\mathrm{e}^{i H t} D \mathrm{e}^{-i H t}$ [9], and Mellin transform estimates [27] to prove Theorem 3 in Sect. 4.

In an Appendix, we prove a result on the invariance of operator domains used in Sect. 2 .

\section{Regularization of the Potential V}

Proposition 1.1. Let $V$ satisfy hypothesis (i) of Theorem 1. Then $V=W+\bar{W}$, where:

(a) $W$ is dilation analytic in $S_{\varphi}$ and $C^{\infty}$ with bounded derivatives,

(b) $\mid \nabla W(x) \leqq C(1+|x|)^{-(1+\alpha)}$, and

(c) $(1+|x|)^{(1+\alpha-\varepsilon)} \bar{W}(x)$ is uniformly locally $L^{2}$ for any $\varepsilon>0$.

Remarks. 1. Conclusion (c) implies that $\left\|\bar{W}\left(\mathrm{H}_{0}+i\right)^{-\gamma} F\left(|x|<R^{1-\eta}\right)\right\|$ is an integrable function of $R$ on $(0, \infty)$ for some $\eta>0$ and $\gamma$ large enough [33, Ex. 2.1]. 2. $\bar{W}$ is obviously $H_{0}$-compact since it is the difference of two $H_{0}$-compact operators.

Proof. We set

$$
W(x)=(4 \pi)^{-n / 2} \int d^{n} y \mathrm{e}^{-(x-y)^{2} / 4} V(y)
$$

( $W$ is the Weierstrass transform of $V$; see $[11,25]$ ). The integral in $(1.1)$ converges absolutely since, by a result of Strichartz [34], any $H_{0}$-bounded multiplication operator is uniformly locally $L^{2} . W$ is obviously $C^{\infty}$ with bounded derivatives by the smoothness and decay of $\exp \left(-(x-y)^{2} / 4\right)$. To see that $W$ is dilation analytic, first note that $W$ is $H_{0}$-compact. For, letting $C=V\left(H_{0}+i\right)^{-1}$ and $T(y)=$ translation by $y$, we can write:

$$
W\left(H_{0}+i\right)^{-1}=(4 \pi)^{-n / 2} \int d^{n} y T(y)^{-1} C T(y) \exp \left(-y^{2} / 4\right) .
$$

The integrand is compact and norm-continuous since $C$ is compact and $T(y)$ is strongly continuous: since the integral converges in operator norm, we conclude that $W\left(H_{0}+i\right)^{-1}$ is compact. Next note that, for real $\theta$,

$$
W(\theta)(x) \equiv W\left(e^{\theta} x\right)=(4 \pi)^{-n / 2} e^{-n \theta / 2} \int d^{n} y V\left(e^{\theta}(x-y)\right) \exp \left\{-e^{-2 \theta} y^{2} / 4\right\},
$$

so that as an operator (again $T(y)$ denotes translation by $y$ ):

$$
W(\theta)=(4 \pi)^{-n / 2} e^{-n \theta / 2} \int d^{n} y T(y)^{-1} V(\theta) T(y) \exp \left\{-e^{-2 \theta} y^{2} / 4\right\} .
$$

Hence if $C(\theta)=V(\theta)\left(H_{0}+i\right)^{-1}$,

$$
W(\theta)\left(H_{0}+i\right)^{-1}=(4 \pi)^{-n / 2} e^{-n \theta / 2} \int d^{n} y T(y)^{-1} C(\theta) T(y) \exp \left\{-e^{-2 \theta} y^{2} / 4\right\} .
$$

Now $C(\theta)$ is an analytic bounded operator valued function in $S_{\varphi}$ and the kernel $\exp \left\{-e^{-2 \theta} y^{2} / 4\right\}$ is analytic in $\theta$ and rapidly decaying for $|\operatorname{Im} \theta|<\pi / 4$, so the integral converges absolutely. The integrand is norm continuous and analytic in $\theta$ : hence $W(\theta)\left(H_{0}+i\right)^{-1}$ extends to an analytic bounded operator valued function in $S_{\varphi}$. This proves (a). 
To prove (b), we estimate:

$$
\begin{aligned}
\left|(1+|x|)^{(1+\alpha)} \nabla W(x)\right| \leqq & (4 \pi)^{-n / 2}(1+|x|)^{(1+\alpha)} \\
& \cdot\left\{\int_{|y|<\frac{|x|}{2}}+\int_{\left.|y|>\frac{|x|}{2}\right\}}\right\}|(\nabla V)(x-y)| e^{-y^{2} / 4} d^{n} y .
\end{aligned}
$$

The first term is bounded since $(1+|x|)^{(1+\alpha)} \nabla V$ is uniformly locally $L^{2}$ and the second is bounded owing to the rapid decay of $\exp \left(-y^{2} / 4\right)$. This gives (b).

Finally, (c) is proved as follows. Pick $\varepsilon>0$. Let $\chi_{c}$ be the characteristic function of the unit cube centered at $c \in \mathbb{Z}^{n}$; we want to show that $\sup \left\|(1+|x|)^{(1+\alpha-\varepsilon)} \bar{W} \chi_{c}\right\|_{2}$ $<\infty$. Write

$$
(1+|x|)^{(1+\alpha-\varepsilon)} \bar{W}=(4 \pi)^{-n / 2}(1+|x|)^{(1+\alpha-\varepsilon)} \int d^{n} y[V(x-y)-V(x)] e^{-y^{2} / 4},
$$

and split the region of $y$-integration into $|y|<|x|^{\delta}$ and $|y|>|x|^{\delta}$ for some $\delta<\varepsilon$. The integral over $|y|>|x|^{\delta}$ decays rapidly in $|x|$. The $L^{2}$ norm of the other term is given by

Write

$$
\left[\int d^{n} x\left(\chi_{c}(1+|x|)^{(1+\alpha-\varepsilon)} \int_{|y|<|x|^{\delta}}[V(x-y)-V(x)] e^{-y^{2} / 4} d^{n} y\right)^{2}\right]^{1 / 2} .
$$

$$
V(x-y)-V(x)=\int_{0}^{1} y \cdot \nabla V(x-t y) d t
$$

true in distributional sense. Putting this in (1.2), we can dominate (1.2) by

$$
|2 c|^{\delta} \sup _{t \in(0,1)} \sup _{|y|<|2 c|^{\delta}}\left(\int\left|\chi_{c}(1+|x|)^{(1+\alpha-\varepsilon)} \nabla V(x-t y)\right|^{2} d^{n} x\right)^{1 / 2} .
$$

Since we have chosen $\delta<\varepsilon$, this is bounded uniformly in $c$.

We note for later use (cf. Sect. 4, especially Lemma 4.2) that, by Hormander's construction ([12], Lemma 3.3), we can further regularize the $C^{\infty}$ potential $W$ :

Proposition 1.2. Let $W$ be a $C^{\infty}$ function with bounded derivatives and suppose that $|\nabla W(x)| \leqq C(1+|x|)^{-(1+\alpha)}$ for some $\alpha>\frac{1}{2}$. Then for any $\delta$ with $0<\delta<\alpha$, we can write $W=W^{\prime}+W^{\prime \prime}$, where

(a) $W^{\prime}$ is $C^{\infty}$ and $\left|\left(D^{\beta} W\right)(x)\right| \leqq C_{|\beta|}(1+|x|)^{-m(|\beta|)}$, where $m(\mathrm{j})=1+j \delta$, $j=1,2, \ldots$

(b) $\left|W^{\prime \prime}(x)\right| \leqq C(1+|x|)^{-(1+\varepsilon)}$ for some $\varepsilon>0$, i.e., $W^{\prime \prime}$ is a short-range potential.

For the proof see ([12], Lemma 3.3).

The operator $H=H_{0}+W$ has several nice domain properties that follow from the smoothness of $W$. We collect them in:

Proposition 1.3. Let $H=H_{0}+W$, where $W$ is $C^{\infty}$ with bounded derivatives. Then

(a) $D\left(H^{\alpha}\right)=D\left(H_{0}^{\alpha}\right)$ for all positive integers $\alpha$,

(b) $\exp (i s H)$ and $(H+i)^{-1}$ preserve $\mathscr{S}\left(\mathbb{R}^{n}\right)$,

(c) For any $g \in C_{0}^{\infty}(\mathbb{R}), g(H)$ preserves $\mathscr{S}\left(\mathbb{R}^{n}\right)$.

Proof. Part (a) follows by calculating the difference $H^{\alpha}-H_{0}^{\alpha}$ in the operator sense on vectors in $\mathscr{S}\left(\mathbb{R}^{n}\right)$. The difference consists of a sum of lower powers of $H_{0}$ times 
derivatives of $W$; such terms are $H_{0}$-bounded and since $\mathscr{S}\left(\mathbb{R}^{n}\right)$ is a core for $H_{0}^{\alpha}$, it follows that $D\left(H^{\alpha}\right)=D\left(H_{0}^{\alpha}\right)$, proving (a). To see that $\exp (i s H)$ preserves $\mathscr{S}\left(\mathbb{R}^{n}\right)$, introduce the seminorms $\|u\|_{k}=\sup \left\|x^{j} H_{0}^{m} u\right\|$ for multi-indices $k$ (these $\stackrel{J \leqq k}{m \leqq|k|-|j|}$

seminorms generate the usual topology on $\mathscr{S}$ ). By a result of Hunziker [13], for any $u \in \mathscr{S}\left(\mathbb{R}^{n}\right)$,

$$
\|\exp (i s H) u\|_{k} \leqq C_{|k|}(1+|s|)^{|k|}\|u\|_{k} .
$$

Hence $\exp (i s H)$ preserves $\mathscr{S}\left(\mathbb{R}^{n}\right) .(H+i)^{-1}$ preserves $\mathscr{S}\left(\mathbb{R}^{n}\right)$ since

$$
(H+i)^{-1}=-i \int_{0}^{\infty} e^{-s} e^{i H s} d s,
$$

so by (1.3),

$$
\left\|(H+i)^{-1} u\right\|_{k} \leqq D_{|k|}\|u\|_{k}
$$

This proves (b). (c) follows similarly by writing

$$
g(H)=(2 \pi)^{-1 / 2} \int_{-\infty}^{+\infty} d s \hat{g}(s) \exp (i s H)
$$

and using the bound (1.3).

\section{The Basic Estimate}

In what follows, we will denote by $\sigma_{\text {p.p. }}(H)$ the pure point spectrum of the operator $H$, i.e., the set of eigenvalues of $H$. We will prove:

Theorem 2.1. Let $H=H_{0}+W$, where $W$ is $C^{\infty}$ with bounded derivatives and dilation analytic in $S_{\varphi}$. Let $g \in C_{0}^{\infty}\left((0, \infty) \backslash \sigma_{\text {p.p. }}(H)\right)$. Then for any positive integer $N$, any $\varepsilon>0$, and any $t$ with $\pm t \in(0, \infty)$,

$$
\left\|F\left(|x| \leqq|t|^{1-\varepsilon}\right) e^{-i t H} g(H) P_{ \pm}\right\| \leqq C_{N, \varepsilon}(1+|t|)^{-N}
$$

Remarks. 1. Since eigenvalues of $H$ accumulate only at 0 , the set of vectors $g(H) \varphi$ with $\varphi \in L^{2}$ and $g$ as above is dense in $\mathscr{H}_{\text {a.c. }}(H)$. 2. Remark 1 and estimate (2.1) imply that $P_{\mp} e^{-i t H} P_{\text {a.c. }}(H) \stackrel{s}{\rightarrow} 0$ as $t \rightarrow \pm \infty$. 3. The proof of Theorem 2.1 depends on the analyticity of $U(\theta) P_{ \pm}=e^{i \theta D} P_{ \pm}$for $\pm \operatorname{Im} \theta>0$. Given an analytic vector $\psi$ for $D$, $e^{i \theta D} \psi$ is a vector-valued analytic function of $\theta$ for $\pm \operatorname{Im} \theta<\delta$ for some $\delta>0$. By mimicking the proof of Theorem 2.1 below, we can show that the estimate

$$
\left\|F\left(|x| \leqq|t|^{1-\varepsilon}\right) e^{-i t H} g(H) \psi\right\| \leqq C_{N, \varepsilon, \psi}(1+|t|)^{-N}
$$

holds for analytic vectors $\psi$ for $D$. The constant $C_{N, \varepsilon, \psi}$ depends on $\psi$ through sup $\left\|e^{-\theta D} \psi\right\|$ for some $\delta^{\prime}<\delta$. $0<\theta<\delta^{\prime}$

Theorem 2.1 follows immediately from:

Theorem 2.2. With the hypothesis and notation of Theorem 2.1, for any positive integer $N$ and $\varepsilon^{\prime}>0$,

$$
\left\|(1+|x|)^{-N} e^{-i t H} g(H) P_{ \pm}\right\| \leqq C_{N, \varepsilon}(1+|t|)^{-N+\varepsilon^{\prime}} .
$$


Our approach to proving Theorem 2.2 follows Mourre's proof of Lemma 1 in [26], where a similar estimate is proven for $H=H_{0}=-\frac{1}{2} \Delta$. To extent his approach to our case, we need Proposition 2.5 below and the results of the Appendix (see Lemma 2.4). For the reader's convenience, we repeat the arguments of [26]. We begin with several reductions.

Lemma 2.3. Suppose that $H, g$ obey the hypotheses of Theorem 2.2, that $\pm t \in(0, \infty)$, and that for every positive integer $N$,

$$
\left\||D+i|^{-(N+2)} e^{-i H t} g(H) P_{ \pm}\right\| \leqq C_{N}(1+|t|)^{-N} .
$$

Then the conclusion of Theorem 2.2 holds.

Proof. By a simple interpolation, (2.3) implies that

$$
\left\||D+i|^{-N} e^{-i H t} g(H) P_{ \pm}\right\| \leqq C_{N, \varepsilon^{\prime}}(1+|t|)^{-N+\varepsilon^{\prime}}
$$

for any $\varepsilon^{\prime}>0$. By writing

$$
(1+|x|)^{-N} e^{-i H t} g(H) P_{ \pm}=(1+|x|)^{-N}(H+i)^{-N} e^{-i H t}\left[(H+i)^{N} g(H)\right] P_{ \pm},
$$

we are reduced to showing that the operator $(1+|x|)^{-N}(H+i)^{-N}|D+i|^{N}$ is bounded. To do this we need only show that terms of the form

$$
(1+|x|)^{-N}(H+i)^{-N} x_{i_{1}} p_{l_{1}} \cdots x_{i_{N}} p_{t_{N}}
$$

are bounded. By Proposition 1.3 (a), $D\left(H_{0}^{N}\right)=D\left(H^{N}\right)$ for all positive integers $N$ and $(H+i)^{-1}$ preserves $\mathscr{S}$. By commutation, one can rewrite (2.4) as a sum of bounded terms plus terms of the form $(1+|x|)^{-N} x_{i_{1}} \cdots x_{i_{N}}(H+i)^{-N} p_{i_{1}} \cdots p_{i_{N}}$. The factor involving the $x_{i_{k}}$ is obviously bounded for all $N$; the factor involving the $p_{i_{k}}$ is bounded for $N$ even and hence for all $N$ by interpolation.

To estimate $\left\||D+i|^{-(N+2)} e^{-i H t} g(H) P_{ \pm}\right\|$, we reexpress $e^{-i H t}$ in terms of the resolvent of $H$ and prove a resolvent bound using the dilation analyticity of $H$. The first step is

Lemma 2.4. (2.3') holds if for any compact subset $K$ of $(0, \infty) \backslash \sigma_{\text {p.p. }}(H)$,

$$
\sup _{\substack{\lambda \in K \\ \varepsilon>0}}\left\||D+i|^{-(N+2)}(H-\lambda \mp i \varepsilon)^{-(N+1)} P_{ \pm}\right\|<\infty .
$$

Proof. We first note that for $\pm t \in(0, \infty), g \in C_{0}^{\infty}$,

$$
e^{-i H t} g(H)=\lim _{\varepsilon \downarrow 0} \frac{N !}{2 \pi i} \frac{(-1)}{(i t)^{N}} \int_{-\infty}^{+\infty} \mathrm{d} \lambda(H-\lambda \mp i \varepsilon)^{-(N+1)} e^{-i \lambda t} g(H) .
$$

(2.6) follows from the functional calculus if we apply the Cauchy integral formula for the $\mathrm{N}^{\text {th }}$ derivative to the function $f_{\varepsilon}(x)=e^{-\varepsilon|t|} e^{-i t x}$. For $f_{\varepsilon}(x) \rightarrow e^{-i t x}$ in sup norm as $\varepsilon \downarrow 0$, and by Cauchy formula

$$
f_{\varepsilon}(x)=\frac{N !}{2 \pi i} \frac{(-1)}{(i t)^{N}} \int_{-\infty}^{+\infty} \mathrm{d} \lambda(x-\lambda \mp i \varepsilon)^{-(N+1)} e^{-i \lambda t}
$$


for $x$ in a fixed compact subset of $\mathbb{R}$. Hence to show that $\left(2.3^{\prime}\right)$ holds, it suffices by (2.6) to show that

$$
\left\||D+i|^{-(N+2)}(H-\lambda \mp i \varepsilon)^{-(N+1)} g(H) P_{ \pm}\right\|
$$

is an integrable function of $\lambda$. Since $g$ has compact support, (2.7) decays rapidly outside any compact subset $K$ of $(0, \infty)$ containing suppg. We can find such a compact $K$ away from eigenvalues of $H$. Hence we need only show that (2.7) is bounded uniformly in $\lambda \in K$ and $\varepsilon>0$. Furthermore, we show in Corollary A. 6 of the Appendix that if $g \in C_{0}^{\infty}$, then $g(H)$ preserves the domain of $D^{N}$ for all positive integers $N$. Hence $|D+i|^{-N} g(H)|D+i|^{N}$ is a bounded operator, so (2.7) is bounded if (2.5) holds.

To prove (2.5) we consider the operator-valued function

$$
F(\theta)=|D+i|^{-(N+2)}(H(\theta)-\lambda \mp i \varepsilon)^{-(N+1)} e^{i \theta D} P_{ \pm},
$$

which by hypothesis extends to an analytic bounded operator-valued function in the strip $0< \pm \operatorname{Im} \theta<\varphi$. We will derive the following differential inequality on its restriction $G(s)=F(i s)$ to the imaginary axis:

$$
\left\|G^{\prime}(s)\right\| \leqq C_{K, \delta}\|G(s)\|^{(N+1) /(N+2)}|s|^{-(N+1) /(N+2)}, 0< \pm s<\delta,
$$

for some positive $\delta<\varphi$ and $C_{K, \delta}$ independent of $\varepsilon$. We can integrate (2.9) directly and conclude that $G(s)$ is uniformly bounded in $(0, \pm \delta)$. In fact, $G(s)$ is Hölder continuous in $s$ ! So it clearly suffices to prove (2.9). We first need an a priori estimate on the resolvent of $H(\theta)$.

Proposition 2.5. Let $K$ be any compact subset of $(0, \infty)$ not containing eigenvalues of $H$. Then there is a $\delta>0$ so that, uniformly in $0< \pm \operatorname{Im} \theta<\delta$,

$$
\sup _{\substack{\lambda \in K \\ \varepsilon>0}}\left\|(H(\theta)-\lambda \mp i \varepsilon)^{-1}\right\| \leqq C_{\mathrm{K}, \delta}|\operatorname{Im} \theta|^{-1} .
$$

Proof. We will show that for any $\lambda_{0} \in(0, \infty) \backslash \sigma_{\text {p.p. }}(H)$, there is some interval $\left(\lambda_{0}-\frac{\eta}{2}, \lambda_{0}+\frac{\eta}{2}\right) \subset(0, \infty) \backslash \sigma_{\text {p.p. }}(H)$ and a $\delta>0$ for which $(2.10)$ holds. The proposition then follows by a covering argument. Further, we will only estimate $\left\|(H(\theta)-\lambda-i \varepsilon)^{-1}\right\|$, since the other estimate follows by taking adjoints. Finally, since $\left(H\left(\theta_{0}+i \theta_{1}\right)-z\right)^{-1}$ and $\left(H\left(i \theta_{1}\right)-z\right)^{-1}$ are unitarily equivalent, we will suppose that $\operatorname{Re} \theta=0$ without loss.

By the spectral theorem, for any $\lambda_{0}>0$ there is a neighborhood $N$ of $\lambda_{0}$ contained in $(0, \infty)$ so that

$$
\left\|\left(e^{-2 i \theta_{1}} H-\lambda-i \varepsilon\right)^{-1}\right\| \leqq C\left|\theta_{1}\right|^{-1}
$$

for $\lambda \in N$, where $C$ is uniform in $\varepsilon>0, \lambda \in N$ and $0<\theta_{1}<\varphi$. Furthermore,

$$
(H(\theta)-z)^{-1}=\left(e^{-2 \theta} H-z\right)^{-1}\left[\mathbb{1}+X(\theta)\left(e^{-2 \theta} H-z\right)^{-1}\right]^{-1},
$$

where

$$
X(\theta)=\left(1-e^{-2 \theta}\right) W+(W(\theta)-W)
$$

whenever

$$
\left\|X(\theta)\left(e^{-2 \theta} H-z\right)^{-1}\right\|<1 .
$$


By the estimate $\left(2.10^{\prime}\right)$, we need only prove that $\left\|X\left(i \theta_{1}\right)\left(e^{-2 i \theta_{1}} H-\lambda-i \varepsilon\right)^{-1}\right\|<1$ uniformly in $0<\theta_{1}<\delta, \lambda \in\left(\lambda_{0}-\eta, \lambda_{0}+\eta\right)$ and $\varepsilon>0$ for some numbers $\delta>0, \eta>0$. Equation (2.11b) and the hypotheses on $W$ show that $X(\theta)=\theta \cdot Y(\theta)$, where $Y(\theta)(H+i)^{-1}$ is an analytic compact operator-valued function of $\theta$. Since there are no eigenvalues of $H$ in a neighborhood of $\lambda_{0}, E_{\left(\lambda_{0}-\eta, \lambda_{0}+\eta\right)}(H) \stackrel{s}{\rightarrow} 0$ as $\eta \rightarrow 0$ so $\left\|Y(\theta) E_{\left(\lambda_{0}-\eta, \lambda_{0}+\eta\right)}(H)\right\| \rightarrow 0$ as $\eta \rightarrow 0$ (by analyticity this holds uniformly for $|\operatorname{Im} \theta|<\varphi / 2)$. So we insert $\mathbb{1}=E_{\left(\lambda_{0}-\eta, \lambda_{0}+\eta\right)}(H)+E_{\mathbb{R} \backslash\left(\lambda_{0}-\eta, \lambda_{0}+\eta\right)}(H)$ in

$$
\begin{aligned}
\left\|X(\theta)\left(e^{-2 \theta} H-\lambda-i \varepsilon\right)^{-1}\right\| \leqq & |\theta|\left\|Y(\theta) E_{\left(\lambda_{0}-\eta, \lambda_{0}+\eta\right)}(H)\right\|\left\|\left(e^{-2 \theta} H-\lambda-i \varepsilon\right)^{-1}\right\| \\
& +|\theta|\left\|Y(\theta)(H+i)^{-1}\right\| \|(H+i)\left(e^{-2 \theta} H-\lambda-i \varepsilon\right)^{-1} \\
& \cdot E_{\mathbb{R} \backslash\left(\lambda_{0}-\eta, \lambda_{0}+\eta\right)}(H) \| .
\end{aligned}
$$

Put $\theta=i \theta_{1}$. In the first term, $|\theta|=\left|\theta_{1}\right|$ cancels the singularity of the resolvent up to a constant factor that can be made small by choosing $\eta$ small enough. If we then restrict $\lambda$ to the interval $\left(\lambda_{0}-\frac{\eta}{2}, \lambda_{0}+\frac{\eta}{2}\right)$, the second term is bounded by a constant times $|\theta|=\left|\theta_{1}\right|$, so it can be made small by restricting $\theta_{1}$ to $0<\theta_{1}<\delta$ for some $\delta>0$. Hence $\left\|X(\theta)\left(e^{-2 i \theta_{1}} H-\lambda-i \varepsilon\right)^{-1}\right\|<1$, uniformly in $\lambda \in\left(\lambda_{0}-\frac{\eta}{2}, \lambda_{0}+\frac{\eta}{2}\right)$, $0<\theta_{1}<\delta$, and $\varepsilon>0$, and the proposition is proved.

To prove the differential inequality (2.9), we note that if $\theta=\theta_{0}+i \theta_{1}$, then by $(2.8)$

$$
F(\theta)=|D+i|^{-(N+2)} e^{i \theta_{0} D}\left(H\left(i \theta_{1}\right)-\lambda \mp i \varepsilon\right)^{-(N+1)} e^{-\theta_{1} D} P_{ \pm} .
$$

Taking the derivative along the real direction, we find

$$
F^{\prime}(\theta)=|D+i|^{-(N+2)} i D e^{i \theta_{0} D}\left(H\left(i \theta_{1}\right)-\lambda \mp i \varepsilon\right)^{-(N+1)} e^{-\theta_{1} D} P_{ \pm} .
$$

But $G^{\prime}(s)=i F^{\prime}(s)$ so we have

$$
\left\|G^{\prime}(s)\right\| \leqq\left\||D+i|^{-(N+1)}\left(H\left(i \theta_{1}\right)-\lambda \mp i \varepsilon\right)^{-(N+1)} e^{-\theta_{1} D} P_{ \pm}\right\| .
$$

To obtain (2.9) we estimate $\|A(z)\|=\left\||D+i|^{-z}(H(i s)-\lambda \mp \mathrm{i} \varepsilon)^{-(N+1)} \cdot e^{-s D} P_{ \pm}\right\|$by interpolating between $\operatorname{Re} z=0$ and $\operatorname{Re} z=N+2$. For $\operatorname{Re} z=0$ we have $\|A(z)\|$ $\leqq C_{K, \delta}|s|^{-(N+1)}$ by Proposition 2.5, while for $\operatorname{Re} z=N+2,\|A(z)\|=\|G(s)\|$. Inequality (2.9) follows since $N+1=0 \cdot \frac{1}{N+2}+(N+2) \cdot \frac{N+1}{N+2}$.

We have thus proven:

Lemma 2.6. The differential inequality (2.9) holds.

Collecting Lemmas 2.6, 2.4, and 2.3, Theorem 2.2 is proved.

\section{Evolution of Observables Under $\exp (-i t H)$}

In this section we use ideas of Enss [9] to study the Heisenberg operators $\mathbf{x}(t), \mathbf{p}(t)$, and $D(t)$, where $A(t) \equiv e^{i H t} A e^{-i H t}$. We will prove: 
Theorem 3.1. Let $H=H_{0}+W$ where $W$ satisfies (i)" of Theorem 3. Then

(a) $\frac{D(t)}{2 t} \rightarrow H$ in strong resolvent sense as $t \rightarrow \pm \infty$ on $\mathscr{H}_{\text {a.c. }}(H)$.

(b) Let $0<\delta<\alpha$. Then $\frac{\mathbf{x}(t)-t \mathbf{p}(t)}{|t|^{1-\delta}} \rightarrow 0$ in strong resolvent sense as $t \rightarrow \pm \infty$ on $\mathscr{H}_{\text {a.c. }}(H)$.

Remark. Theorem 3.1 (a) and its proof below are due to Enss [9]; Theorem 3.1 (b) is new.

Theorem 3.1 implies:

Theorem 3.2. Let $\psi \in \mathscr{H}_{\text {a.c. }}(H)$ and suppose that $\psi=E_{(a, b)}(H) \psi$, where $(a, b) \subset(0, \infty) \backslash \sigma_{\text {p.p. }}(H)$. Let $\psi_{t}=e^{-i t H} \psi$. Then:

$$
\psi_{t}-F_{0}\left(|\mathbf{x}-\mathbf{p} t|<|t|^{1-\delta}\right) E_{(a, b)}\left(\frac{D}{2 t}\right) g\left(H_{0}\right) \psi_{t} \rightarrow 0 \text { as } t \rightarrow \pm \infty,
$$

where $g \in C_{0}^{\infty}(0, \infty)$ satisfies $g=1$ on $(a, b)$.

Remark. For an $n$-tuple A of commuting self-adjoint operators and a subset $S$ of $\mathbb{R}^{n}$, the "smooth" projection $F_{0}(\mathbf{A} \in S)$ is defined as follows. Let $\chi_{S}$ denote the characteristic function of $S$ and let $\xi$ satisfy $S d^{n} y \xi(y)=1$ and $\hat{\xi} \in C_{0}^{\infty}\left(\mathbb{R}^{n}\right) . F_{0}(\mathbf{A} \in S)$ is the operator associated to the convolution $\chi_{S}{ }^{*} \xi$ by the functional calculus for $\mathbf{A}$.

Proof of Theorem 3.2 given Theorem 3.1. It is enough to show that $\psi_{t}-E_{(a, b)}\left(\frac{D}{2 t}\right) \psi_{t} \rightarrow 0$ as $t \rightarrow \pm \infty$ and $\psi_{t}-F_{0}\left(|\mathbf{x}-\mathbf{p} t|<|t|^{1-\delta}\right) \psi_{t} \rightarrow 0$ as $t \rightarrow \pm \infty$ separately, since the result then follows by the uniform boundedness in $t$ of the projections. By Theorem 3.1 (a) and Theorem VIII. 24(b) of [28],

$$
E_{(a, b)}\left(\frac{D(t)}{2 t}\right) \stackrel{s}{\rightarrow} E_{(a, b)}(H)
$$

as $t \rightarrow \pm \infty$. Write

$$
\begin{aligned}
\left\|\psi_{t}-E_{(a, b)}\left(\frac{D}{2 t}\right) \psi_{t}\right\| & =\left\|\left[E_{(a, b)}(H)-E_{(a, b)}\left(\frac{D}{2 t}\right)\right] \psi_{t}\right\| \\
& =\left\|\left[E_{(a, b)}(H)-E_{(a, b)}\left(\frac{D(t)}{2 t}\right)\right] \psi\right\| \rightarrow 0,
\end{aligned}
$$

where in the last step we have used the unitarity of $e^{i t H}$. A similar argument using Theorem 3.1 (b) shows that $\|\left(1-F_{0}\left(|x-p t|<|t|^{1-\delta}\right) \psi_{t} \| \rightarrow 0\right.$. Finally, since $g=1$ on $(a, b),\left(1-g\left(H_{0}\right)\right) \psi_{t}=\left(g(H)-g\left(H_{0}\right)\right) \psi_{t}$, which goes to zero by the compactness of $(H+i)^{-1}-\left(H_{0}+i\right)^{-1}$ and a standard argument [33, Lemma 2.4].

To prove Theorem 3.1, we first recall a standard criterion [28, Theorem VIII. 25 (a) ] for strong resolvent convergence: $A_{n} \rightarrow A$ in strong resolvent sense if $A_{n} \rightarrow A$ on a core for $A$ contained in $D\left(A_{n}\right)$ for each $n$. Hence our first step is to find a nice set of vectors on which to study the Heisenberg operators $D(t)$ and $\mathbf{x}(t)-t \mathbf{p}(t)$. 
Proposition 3.3. Let $N=p^{2}+x^{2}+1$ and let $\mathscr{D}$ be the set of all vectors of the form $g(H) e^{-\theta N} \varphi$ for $\varphi \in L^{2}, \theta>0$, and $g \in C_{0}^{\infty}\left((0, \infty) \backslash \sigma_{\text {p.p. }}(H)\right)$. Then:

(a) $\mathscr{D}$ is a core for $H$ 个 $\mathscr{H}_{\text {a.c. }}(H)$.

(b) $\mathscr{D} \subset \mathscr{S}\left(\mathbb{R}^{n}\right)$ and $\exp (-i t H) \mathscr{D} \subset \mathscr{S}\left(\mathbb{R}^{n}\right)$.

(c) $\left\|F\left(|x|<|t|^{1-\varepsilon}\right) e^{-i t H} \psi\right\| \leqq C_{N}(1+|t|)^{-N}$ for any $\varepsilon>0, \mathrm{t} \in \mathbb{R}$, and $\psi \in \mathscr{D}$.

Proof. Since $e^{-\theta N} \psi \rightarrow \psi$ as $\theta \rightarrow 0$, $\mathscr{D}$ is dense in $\bigcup_{g} \operatorname{ran} g(H)$, which is obviously dense in $D\left(H \uparrow \mathscr{H}_{\text {a.c. }}(H)\right)$ in graph norm, proving (a). (b) holds since for $\theta>0$, $e^{-\theta N} \psi \in C^{\infty}(N)=\mathscr{S}\left(\mathbb{R}^{n}\right)$ [28] and by Proposition 1.3, $g(H)$ and $\exp (-i t H)$ both preserve $\mathscr{S}\left(\mathbb{R}^{n}\right)$. Finally (c) holds since, for $\theta>0, e^{-\theta N} \psi$ is an analytic vector for $N$ and $N$ analytically dominates $D$ (e.g. by Faris [10, Theorem 16.4]); hence any $\psi \in \mathscr{D}$ is of the form $g(H) \chi$ where $\chi$ is an analytic vector for $D$, and by Remark 3 after Theorem 2.1, such vectors obey the estimate of (c).

Proof of Theorem 3.1. Following the method of [9], we consider the Heisenberg equations of motion for $D(t)$ and $\mathbf{x}(t)-\mathbf{p}(t) \cdot t$. Weakly on $\mathscr{D} \times \mathscr{D}$,

$$
\frac{d}{d t} D(t)=e^{i H t}\left[2 H_{0}-(x \cdot \nabla) W\right] e^{-i H t},
$$

and by Proposition 3.3(b), (3.1) holds in the operator sense on $\mathscr{D}$. Write the quantity in brackets as $2 H+I$; by Proposition 1.1, $I$ is $H$-compact. Integrate (3.1) and divide by $2 t$ to obtain

$$
\frac{D(t)}{2 t}=\frac{D(0)}{2 t}+H+\frac{1}{2 t} \int_{0}^{t} d s e^{i s H} I e^{-i s H} .
$$

Applied to vectors $\psi \in \mathscr{D}$, the first term in $\left(3.1^{\prime}\right)$ vanishes by the RAGE theorem [29, Theorem XI.115] since $I(H+i)^{-1}$ is compact and $(H+i) \psi$ is bounded, if $\psi \in \mathscr{D}$. This proves (a). To prove (b) we compute, weakly on $\mathscr{D} \times \mathscr{D}$,

$$
\frac{d}{d t}(\mathbf{x}(t)-t \cdot \mathbf{p}(t))=e^{i t H}(\nabla W)(x) e^{-t t H} .
$$

Again, (3.2) actually holds in the operator sense on $\mathscr{D}$. Integrate (3.2) and divide by $|t|^{1-\delta}$ to obtain

$$
\frac{\mathbf{x}(t)-t \cdot \mathbf{p}(t)}{|t|^{1-\delta}}=\frac{\mathbf{x}(0)}{|t|^{1-\delta}}+\frac{1}{|t|^{1-\delta}} \int_{0}^{t} e^{i t H}(\nabla W)(x) e^{-i t H} d s .
$$

The first term vanishes as $t \rightarrow \pm \infty$ when applied to $\psi \in \mathscr{D}$. The integrand of the second term applied to $\psi \in \mathscr{D}$ is estimated using Proposition 3.3(c) and the estimate on $\nabla W$ in Proposition 1.1 (b): $\left\|(\nabla W)(x) e^{-i s H} \psi\right\| \leqq$ cst. $\times\left\|F\left(|x|<|s|^{1-\varepsilon}\right) e^{-\imath s H} \psi\right\|+\left\|(\nabla W)(x) F\left(|x| \leqq|s|^{1-\varepsilon}\right)\right\|$

$$
\leqq C_{N}(1+|s|)^{-N}+\operatorname{const}(1+|s|)^{-(1+\alpha-\varepsilon)}
$$

for any $\varepsilon>0$. On integrating the right hand side of (3.3) and dividing by $|t|^{1-\varepsilon}$, we obtain an estimate for the second term in $\left(3.2^{\prime}\right)$ that vanishes as $t \rightarrow \pm \infty$, since $\varepsilon>0$ is arbitrary and $\delta<\alpha$. Hence $\frac{\mathbf{x}(t)-\mathbf{p}(t) \cdot t}{|t|^{1-\delta}} \rightarrow 0$ on $\mathscr{D}$, proving (b). 


\section{Proof of Theorem 3}

To prove Theorem 3, we will show that the inverse modified wave operators $\Omega_{D}^{ \pm}\left(H, H_{0}\right)^{*}$ exist as strong limits on a dense subset of $\mathscr{H}_{\text {a.c. }}(H)$. We will only give the proof for $\Omega_{D}^{-*}$ since the proof for $\Omega_{D}^{+*}$ is similar. $\Omega_{D}^{-*}$ exists if

$$
\lim _{s \rightarrow \infty} \sup _{t \geqq s}\left\|\left[e^{-i H(t-s)}-\mathscr{U}_{0}(t, s)\right] e^{-i H s} \psi\right\|=0,
$$

for $\psi$ in a dense subset of $\mathscr{H}_{\text {a.c. }}(H)$. Consider the set of vectors $\psi$ with $E_{(a, b)}(H) \psi=\psi$ for some $(a, b) \subset(0, \infty) \backslash \sigma_{\text {p.p. }}(H)$. For such $\psi,(4.1)$ holds if

$$
\lim _{s \rightarrow \infty} \sup _{t \geqq s}\left\|\left[e^{-i H(t-s)}-\mathscr{U}_{0}(t, s)\right] F_{0}\left(|\mathbf{x}-\mathbf{p} s|<|s|^{1-\delta}\right) g\left(H_{0}\right) E_{(a, b)}\left(\frac{D}{2 s}\right)\right\|=0,
$$

where $g \in C_{0}^{\infty}(0, \infty)$ satisfies $g=1$ on $(a, b)$, by Theorem 3.2. By a "Cook's method" argument, (4.2) holds if

$$
\begin{aligned}
& \lim _{s \rightarrow \infty} \sup _{t \geqq s} \int_{s}^{t} \|\left[W^{\prime}(\mathbf{x})+W^{\prime \prime}(\mathbf{x})-W^{\prime}\left(\mathbf{p} s^{\prime}\right)\right] \mathscr{U}_{0}\left(s^{\prime}, s\right) \\
& \cdot F_{0}\left(|\mathbf{x}-\mathbf{p} s|<|s|^{1-\delta}\right) g\left(H_{0}\right) E_{(a, b)}\left(\frac{D}{2 s}\right) \| d s^{\prime}=0 .
\end{aligned}
$$

We will prove (4.3). We first collect some estimates on the modified free evolution $\mathscr{U}_{0}\left(s^{\prime}, s\right)$. The modified free evolution is dominated by the free evolution $e^{-i H_{0}\left(s^{\prime}-s\right)}$; the first Lemma is a simple extension of the estimate on $e^{-i H_{0} t}$ proven in [27] by Mellin transform methods.

Lemma 4.1.Let $g \in C_{0}^{\infty}(0, \infty)$. Then there is a $c>0$ so that, for all $s^{\prime}$ with $s^{\prime}>s>0$ and any integer $N$,

$$
\left\|F\left(|x|<c s^{\prime}\right) e^{-i\left(s^{\prime}-s\right) \mathrm{H}_{0}} g\left(H_{0}\right) E_{(a, b)}\left(\frac{D}{2 s}\right)\right\| \leqq C_{\mathrm{N}}\left(1+\left|s^{\prime}\right|\right)^{-N} .
$$

We omit the proof.

The next lemma shows that the corrections to the free evolution introduced by the factor $\exp \left[-i \int_{s}^{s^{\prime}} W^{\prime}(\mathbf{p} \tau) \mathrm{d} \tau\right]$ are small.

Lemma 4.2 [8]. Let $\bar{g} \in C_{0}^{\infty}(0, \infty)$ and let $K\left(s^{\prime}, s\right)=\exp \left[-i \int_{s}^{s^{\prime}} W^{\prime}(\mathbf{p} \tau) d \tau\right] \bar{g}\left(H_{0}\right)$. Let $S_{1}, S_{2}$ be subsets of $\mathbb{R}^{n}$ with $\operatorname{dist}\left(S_{1}, S_{2}\right) \geqq d>0$. Then for any integer $l$,

(a) $\left\|F\left(\mathbf{x} \in S_{1}\right) K\left(s^{\prime}, s\right) F\left(\mathbf{x} \in S_{2}\right)\right\| \leqq D_{l}\left(1+\left|s^{\prime}\right|\right)^{\left(1-\delta^{\prime}\right)(n+l+1)}(1+d)^{-l}$, where $\delta^{\prime}$ is defined in Proposition 1.2.

(b) The same estimate holds with $\mathbf{x}$ replaced by $\mathbf{x}-\mathbf{p} s^{\prime}$.

(c) (a) and (b) hold with $F$ replaced by $F_{0}$, where $F_{0}$ is defined as in the remark after Theorem 3.2 .

Proof. (b) follows from (a) since $\exp \left(-i s^{\prime} H_{0}\right)$ commutes with $K\left(\mathrm{~s}^{\prime}, s\right)$ and $\exp \left(-i s^{\prime} H_{0}\right) f(\mathbf{x})=f\left(\mathbf{x}-\mathbf{p} s^{\prime}\right) \exp \left(-i s^{\prime} H_{0}\right)$ for Borel functions $f$. (c) follows from (a) (respectively (b)) by using the rapid decay of $F_{0}(\mathbf{x} \in S)$ [respectively 
$\left.F_{0}(\mathbf{x}-\mathbf{p} s \in S)\right]$ outside of $S$. To prove (a), we note that in $x$-space, $K\left(s^{\prime}, s\right)$ is the operator of convolution with a rapidly decaying kernel. (a) then follows by using the estimates of Proposition 1.2 on derivatives of $W^{\prime}$ together with Young's inequality (cf. [8, Eq. (41) ff.]).

Next, we note some useful properties of the "smooth" projections $F_{0}$ introduced in Sect. 3 .

Lemma 4.3 [8]. Let $F_{0}(\mathbf{x}-\mathbf{p} s \in S)$ be defined as in Sect. 3 .

(a) (small momentum transfer) Let $g \in C_{0}^{\infty}(0, \infty)$. Then for supp $\hat{\xi}$ small enough there is a $\bar{g} \in C_{0}^{\infty}(0, \infty)$ with $\bar{g}=1$ on supp $g$ so that $F_{0}(\mathbf{x}-\mathbf{p} s \in S) g\left(H_{0}\right)$ $=\bar{g}\left(H_{0}\right) F_{0}(\mathbf{x}-\mathbf{p} s \in S) g\left(H_{0}\right)$ and similarly for $s$ replaced by $s^{\prime}$.

(b) (small position transfer) For $c>0$ and supp $\hat{\xi}$ small enough,

$$
\mathrm{F}\left(|x|<\frac{c s^{\prime}}{2}\right) F_{0}\left(\mathbf{x}-\mathbf{p} s^{\prime} \in S\right) F\left(|x|>c s^{\prime}\right)=0 .
$$

Proof. Let $f_{S}=\chi_{S}^{*} \xi$. Then

$$
F_{0}(\mathbf{x}-\mathbf{p} s \in S)=\int d^{n} \lambda \hat{f_{S}}(\lambda) \exp [i \lambda \cdot(\mathbf{x}-\mathbf{p} s)] .
$$

By the Baker-Campbell-Hausdorff formula, $\exp [i \lambda \cdot(\mathbf{x}-\mathbf{p} s)]=[\exp i \lambda \cdot \mathbf{x}] \times[\exp$ $-i \lambda \cdot \mathbf{p} s] \times \exp \frac{1}{2} i \lambda^{2} s$. Using this fact along with the compact support of $\hat{f_{S}}$, it follows that $F_{0}(\mathbf{x}-\mathbf{p} s \in S)$ has a momentum transfer of at most a where $\operatorname{supp} \xi \subset\{\lambda:|\lambda|<a\}$. This shows (a). (b) is proved similarly.

Next we note a formula for the operator difference $W^{\prime}(\mathbf{x})-W^{\prime}\left(\mathbf{p} s^{\prime}\right)$ that occurs in (4.3).

\section{Lemma 4.4.}

$$
W^{\prime}(\mathbf{x})-W^{\prime}\left(\mathbf{p} s^{\prime}\right)=\int_{0}^{1} d \theta\left\{\left(\nabla W^{\prime}\right)\left(\theta \mathbf{x}+(1-\theta) \mathbf{p} s^{\prime}\right) \cdot\left(\mathbf{x}-\mathbf{p} s^{\prime}\right)+i s^{\prime}\left(\Delta W^{\prime}\right)\left(\theta \mathbf{x}+(1-\theta) \mathbf{p} s^{\prime}\right)\right\} .
$$

Lemma 4.4 is proved by writing $W^{\prime}$ as the integral of its Fourier transform and using the formula

$$
e^{i q \cdot \mathbf{x}}-e^{i q \cdot \mathbf{p} s^{\prime}}=i \int_{0}^{1} d \theta e^{i \theta q \cdot \mathbf{x}} i q \cdot\left(\mathbf{x}-\mathbf{p} s^{\prime}\right) e^{i(1-\theta) \mathbf{q} \cdot \mathbf{p} s^{\prime}}
$$

together with the Baker-Campbell-Hausdorff formula. We omit details. Finally, we note:

Lemma 4.5. For any positive integer $k$ and $s^{\prime}>0$,

$$
\sup _{\theta \in[0,1]}\left\|F\left(\left|\theta \mathbf{x}+(1-\theta) \mathbf{p} s^{\prime}\right|<\frac{c s^{\prime}}{8}\right) F_{0}\left(\left|\mathbf{x}-\mathbf{p} s^{\prime}\right|<2 s^{\prime 1-\delta}\right) F\left(|\mathbf{x}|>\frac{c s^{\prime}}{4}\right)\right\|<C_{k}\left(1+s^{\prime}\right)^{-k} \text {. }
$$

The proof is very similar to the proof of Lemma 1 in [8] and is omitted.

We now carry out the

Proof of Theorem 3. We will show that the integrand of (4.3) is estimated by $C\left(1+s^{\prime}\right)^{-(1+\eta)}$ for some $\eta>0$ and $C$ independent of $s, s^{\prime}$. We first note that, by Lemma 4.3, $\quad F_{0}\left(|\mathbf{x}-\mathbf{p} s|<|s|^{1-\delta}\right) g\left(H_{0}\right)=\bar{g}\left(H_{0}\right) F_{0}\left(|\mathbf{x}-\mathbf{p} s|<|s|^{1-\delta}\right) g\left(H_{0}\right)$ for 
some $\bar{g} \in C_{0}^{\infty}(0, \infty)$. Writing $B=W^{\prime}(x)+W^{\prime \prime}(x)-W^{\prime}\left(\mathbf{p} s^{\prime}\right)$, we see that the integrand of (4.3) equals

$$
\left\|B K\left(s^{\prime}, s\right) e^{-\imath\left(s^{\prime}-s\right) H_{0}} F_{0}\left(|\mathbf{x}-\mathbf{p} s|<|s|^{1-\delta}\right) g\left(H_{0}\right) E_{(a, b)}\left(\frac{D}{2 s}\right)\right\|
$$

which, by the identity

$$
e^{-i\left(s^{\prime}-s\right) H_{0}} f(\mathbf{x}-\mathbf{p} s)=f\left(\mathbf{x}-\mathbf{p} s^{\prime}\right) e^{-i\left(s^{\prime}-s\right) H_{0}},
$$

equals

$$
\left\|B K\left(s^{\prime}, s\right) F_{0}\left(\left|\mathbf{x}-\mathbf{p} s^{\prime}\right|<|s|^{1-\delta}\right) e^{-i\left(s^{\prime}-s\right) H_{0}} g\left(H_{0}\right) E_{(a, b)}\left(\frac{D}{2 s}\right)\right\| .
$$

Writing $\mathbb{1}=F\left(|x|<c s^{\prime}\right)+F\left(|x|>c s^{\prime}\right)$ and using Lemma 4.1, we conclude that (4.4) is estimated by a term decaying rapidly in $s^{\prime}$ plus

$$
\left\|B K\left(s^{\prime}, s\right) F_{0}\left(\left|\mathbf{x}-\mathbf{p} s^{\prime}\right|<|s|^{1-\delta}\right) F\left(|\mathbf{x}|>c s^{\prime}\right)\right\| .
$$

By choosing $\delta^{\prime}<\delta$ in Proposition 1.2 ( $\operatorname{so} \frac{1}{2}<\delta^{\prime}<\delta<\alpha$ ), we can use Lemma 4.2 to see that $\left\|F_{0}\left(\left|\mathbf{x}-\mathbf{p} s^{\prime}\right|>2 s^{\prime 1-\delta}\right) K\left(s^{\prime}, s\right) F_{0}\left(\left|\mathbf{x}-\mathbf{p} s^{\prime}\right|<s^{1-\delta}\right)\right\|$ decays rapidly in $s^{\prime}$ for $s^{\prime}>s$. Hence we can estimate (4.5) by terms that decay rapidly in $s^{\prime}$ plus

$$
\left\|B F_{0}\left(\left|\mathbf{x}-\mathbf{p} s^{\prime}\right|<2 s^{\prime 1-\delta}\right) K\left(s^{\prime}, s\right) F_{0}\left(\left|\mathbf{x}-\mathbf{p} s^{\prime}\right|<s^{1-\delta}\right) F\left(|\mathbf{x}|>c s^{\prime}\right)\right\| .
$$

By Lemma 4.3,

$$
F_{0}\left(\left|\mathbf{x}-\mathbf{p} s^{\prime}\right|<s^{\prime 1-\delta}\right) F\left(|\mathbf{x}|>c s^{\prime}\right)=F\left(|\mathbf{x}|>\frac{c s^{\prime}}{2}\right) F_{0}\left(\left|\mathbf{x}-\mathbf{p} s^{\prime}\right|<s^{\prime 1-\delta}\right) F\left(|\mathbf{x}|>c s^{\prime}\right)
$$

for supp $\hat{\xi}$ small enough, and by Lemma 4.2, $\left\|F\left(|\mathbf{x}|<\frac{c s^{\prime}}{4}\right) K\left(s^{\prime}, s\right) F\left(|\mathbf{x}|>\frac{c s^{\prime}}{2}\right)\right\|$ decays rapidly in $s^{\prime}$. Hence, finally, we can dominate (4.6) by terms decaying rapidly in $s^{\prime}$ plus

$$
\begin{aligned}
& \left\|B F_{0}\left(\left|\mathbf{x}-\mathbf{p} s^{\prime}\right|<2 s^{1-\delta}\right) F\left(|\mathbf{x}|>\frac{c s^{\prime}}{4}\right)\right\| \\
& \leqq\left\|\left[W^{\prime}(\mathbf{x})-W^{\prime}\left(\mathbf{p} s^{\prime}\right)\right] F_{0}\left(\left|\mathbf{x}-\mathbf{p} s^{\prime}\right|<2 s^{\prime 1-\delta}\right) F\left(|\mathbf{x}|>\frac{c s^{\prime}}{4}\right)\right\| \\
& \quad+\left\|W^{\prime \prime}(\mathbf{x}) F_{0}\left(\left|\mathbf{x}-\mathbf{p} s^{\prime}\right|<2 s^{\prime 1-\delta}\right) F\left(|\mathbf{x}|>\frac{c s^{\prime}}{4}\right)\right\| .
\end{aligned}
$$

To estimate the first term on the right hand side of (4.7), we use Lemmas 4.4 and 4.5 and bound it by rapidly decaying terms plus

$$
\begin{aligned}
\sup _{\theta \in[0,1]}\{ & \left\|\nabla W^{\prime}\left(\theta \mathbf{x}+(1-\theta) \mathbf{p} s^{\prime}\right) F\left(\left|\theta \mathbf{x}+(1-\theta) \mathbf{p} s^{\prime}\right| \leqq \frac{c s^{\prime}}{8}\right)\right\| \\
& \cdot\left\|\left(\mathbf{x}-\mathbf{p} s^{\prime}\right) F_{0}\left(\left|\mathbf{x}-\mathbf{p} s^{\prime}\right|<2 s^{\prime 1-\delta}\right)\right\| \\
& \left.+s^{\prime}\left\|\Delta W\left(\theta \mathbf{x}+(1-\theta) \mathbf{p} s^{\prime}\right) F\left(\left|\theta \mathbf{x}+(1-\theta) \mathbf{p} s^{\prime}\right|>\frac{c s^{\prime}}{8}\right)\right\|\right\} .
\end{aligned}
$$


The first term in brackets is bounded by a constant times $\left(1+s^{\prime}\right)^{-\left(1+\delta^{\prime}\right)}\left(1+s^{\prime}\right)^{1-\delta}$ $=\left(1+s^{\prime}\right)^{-(1+\eta)}$ for $\eta>0$, since $\delta, \delta^{\prime}>\frac{1}{2}$. The second term in brackets is bounded by a constant times $\left(1+s^{\prime}\right)^{-2 \delta^{\prime}}$, and since $\delta^{\prime}>\frac{1}{2}, 2 \delta^{\prime}=1+\eta$ for some $\eta>0$. The second term in (4.7) is dominated by $\left\|W^{\prime}(\mathbf{x}) F\left(|\mathbf{x}|>\frac{c s^{\prime}}{8}\right)\right\|$ since, by Lemma 4.3,

$$
\begin{aligned}
& F_{0}\left(\left|\mathbf{x}-\mathbf{p} s^{\prime}\right|<2 s^{\prime 1-\delta}\right) F\left(|\mathbf{x}|>\frac{c s^{\prime}}{4}\right) \\
& \quad=F\left(|\mathbf{x}|>\frac{c s^{\prime}}{8}\right) F_{0}\left(\left|\mathbf{x}-\mathbf{p} s^{\prime}\right|>2 s^{\prime 1-\delta}\right) F\left(|\mathbf{x}|>\frac{c s^{\prime}}{4}\right)
\end{aligned}
$$

for suitable choice of $\operatorname{supp} \xi$. $\left\|W^{\prime \prime}(\mathbf{x}) F\left(|\mathbf{x}|>\frac{c s^{\prime}}{8}\right)\right\|$ is estimated by a constant times $\left(1+s^{\prime}\right)^{-(1+\eta)}$ for some $\eta>0$ by Proposition 1.2(b). This shows that (4.3) holds, proving Theorem 3 .

\section{Appendix. On the Invariance of Operator Domains}

Let $A$ be a self-adjoint operator and let $U(\alpha)=\exp (i \alpha A)$. For any self adjoint operator $B, U(\alpha)$ induces a family of self-adjoint operators $B(\alpha)=U(\alpha) B U(\alpha)^{-1}$ unitarily equivalent to $B$. We wantt to show that if the map $\alpha \rightarrow B(\alpha)$ is smooth, nice functions of $B$ preserve $D\left(A^{k}\right), k=1, \ldots, n$, where $n$ depends on the smoothness of the map $\alpha \rightarrow B(\alpha)$. The following Proposition is central:

Proposition A.1. $\varphi \in D\left(A^{k}\right)$ if and only if the vector-valued function $\varphi(\alpha)=U(\alpha) \varphi$ is $C^{k}$ at 0 .

Remark. If $\varphi(\alpha)$ is differentiable at 0 , then by translating with the unitary group, it is differentiable everywhere. Hence the phrase " $C^{k}$ at 0 " makes sense.

Proof. For $k=1$, this is Theorem VIII. 7 of [28]. Suppose the proposition holds for $\varphi \in D\left(A^{k-1}\right)$. If $\varphi(\alpha)$ is $C^{k}$, certainly $\varphi \in D\left(A^{k-1}\right)$ and $\varphi^{(k)}(0)=\psi^{\prime}(0)$, where $\psi$ $=A^{k-1} \varphi$. But then $\psi \in D(A)$, i.e., $\varphi \in D\left(A^{k}\right)$. By a similar argument, any $\varphi \in D\left(A^{k}\right)$ is $C^{k}$ at zero.

Suppose $g$ is a smooth function; then $g(B) \varphi \in D\left(A^{k}\right)$ if $U(\alpha) g(B) \varphi$ is $C^{k}$ at 0 . But $U(\alpha) g(B) \varphi=g(B(\alpha)) U(\alpha) \varphi$ so $U(\alpha) g(B) \varphi$ is $C^{k}$, if $\varphi \in D\left(A^{k}\right)$ and $g(B(\alpha))$ is norm $C^{k}$ as a function of $\alpha$. Hence:

Corollary A.2. Suppose that $B(\alpha)=U(\alpha) B U(\alpha)^{-1}$ as above and that $g(B(\alpha))$ is norm$C^{n}$ as a function of $\alpha$. Then $g(B(\alpha))$ preserves $D\left(A^{k}\right)$ for $k=1, \ldots, n$.

We now find sufficient conditions on the map $\alpha \rightarrow B(\alpha)$ and the function $g$ for $g(B(\alpha))$ to be norm- $C^{n}$. We first consider bounded operators $B$ and then make an easy extension to semibounded self-adjoint operators.

Since the operator $B(\alpha)$ are self-adjoint, we can write

$$
g(B(\alpha))=(2 \pi)^{-1 / 2} \int \hat{g}(t) \exp (i t B(\alpha)) d t .
$$

We are then motivated to consider the operator $\exp i t B(\alpha)$ : 
Lemma A.3. Let $B(\alpha)$ be a family of bounded, self-adjoint operators and suppose that the map $\alpha \rightarrow B(\alpha)$ is norm- $C^{k}$ in some interval I containing 0 . Let $\|B(\alpha)\|_{k}=\sup _{\substack{0 \leq j \leq k \\ \alpha \in I}}\left\|\left(D_{\alpha}^{j} B\right)(\alpha)\right\|$. Then

$$
\|\exp (i t B(\alpha))\|_{k} \leqq C_{k}(1+|t|)^{k}\|B(\alpha)\|_{k} .
$$

Proof. By the Duhamel formula,

$$
\begin{aligned}
\exp i t B(\alpha+\varepsilon)-\exp i t B(\alpha)= & i t \int_{0}^{1} d s \exp (\text { ist } B(\alpha+\varepsilon)) \\
& \cdot[B(\alpha+\varepsilon)-B(\alpha)] \times \exp i(1-s) t \mathrm{~B}(\alpha),
\end{aligned}
$$

so expit $B(\alpha)$ is norm-continuous in $\alpha$ for fixed $t$. Dividing by $\varepsilon$ and taking norm limits, we get

$$
\frac{d}{d \alpha}(\exp i t B(\alpha))=i t \int_{0}^{1} d s \exp (i s t B(\alpha)) B^{\prime}(\alpha) \exp (i(1-s) t B(\alpha)) .
$$

Repeated application of this formula gives (A.2).

Combining (A.1.) and LemmaA.3, and using Corollary A.2, we obviously have:

Proposition A.4. Let $\alpha \rightarrow B(\alpha)$ be norm- $C^{n}$ and let $g \in C_{0}^{\infty}$. Then $g(B(\alpha))$ is norm- $C^{n}$. If $B(\alpha)=U(\alpha) B U(\alpha)^{-1}$ with $B$ and $U(\alpha)$ as above, then $g(B(\alpha))$ preserves $D\left(A^{k}\right)$ for $k=1, \ldots, n$.

Now let $B$ be a semibounded self-adjoint operator and let $B(\alpha)=U(\alpha) B U(\alpha)^{-1}$. Suppose that for some suitable $c, R(\alpha)=(B(\alpha)+c)^{-1}$ is norm- $C^{n}$. If $g \in C_{0}^{\infty}$ $(-c, \infty), f(y)=g\left(\frac{1}{y}-c\right)$ is a $C_{0}^{\infty}$ function so $g(B(\alpha))=f(R(\alpha))$ is norm- $C^{n}$. We have therefore proved:

Theorem A.5. Let $B(\alpha)=U(\alpha) B U(\alpha)^{-1}$, where $B$ is a semibounded self-adjoint operator and $U(\alpha)$ is a unitary group generated by the self-adjoint operator $A$. Suppose that $R(\alpha)=(B(\alpha)+c)^{-1}$ is norm- $C^{n}$ for suitable $c$. Then for any $g \in C_{0}^{\infty}(-c, \infty)$, $g(B(\alpha))$ preserves $D\left(A^{k}\right), k=1, \ldots, n$.

If $H=H_{0}+W$ with $W$ dilation analytic and $H(\theta)=\mathscr{U}(\theta) H \mathscr{U}(\theta)^{-1}, R(\theta)$ $=(H(\theta)+c)^{-1}$ is analytic. Clearly:

Theorem A.6. Let $H=H_{0}+W$ with $W$ dilation analytic and $H+c>0$ for some $c$. Let $g \in C_{0}^{\infty}(-c, \infty)$. Then $g(H)$ preserves the domain of $D^{n}$ for all positive integers $n$.

Acknowledgements. I am grateful to Barry Simon for encouragement and for very helpful discussions, to Volker Enss for helpful discussions, and to the referee for helpful comments. I also thank the Department of Mathematics, California Institute of Technology, for hospitality during part of the time this work was done.

\section{References}

1. Agmon, S.: Some new results in spectral and scattering theory of differential operators on $L^{2}\left(\mathbb{R}^{n}\right)$. Séminaire Goulaouic-Schwartz 1978-9, Centre de Mathématiques-Polytechnique, Palaiseau.

2. Aguilar, J., Combes, J. M.: Commun. Math. Phys. 22, 269-279 (1971) 
3. Alsholm, P., Kato, T.: Scattering with long-range potentials. In: Partial differential equations, pp. 393-399. Proc. Symp. Pure Math., No. 23. Providence, R. I.: Am. Math. Soc. 1973

4. Babbit, D., Balslev, E.: J. Func. Anal. 18, 1-14 (1975)

5. Buslaev, V.S., Matveev, V. B.: Theoret. and Math. Phys. 2, 266-274 (1970)

6. Dollard, J. D.: J. Math. Phys. 5, 729-738 (1964)

7. Enss, V.: Commun. Math. Phys. 61, 285-291 (1978)

8. Enss, V.: Ann. Phys. (N.Y.) 119, 117-132 (1979)

9. Enss, V.: Paper in preparation and "Geometric methods in spectral and scattering theory of Schrödinger operators", Sect. 7. In: Rigorous atomic and molecular physics, eds. G. Velo, A. Wightman. New York: Plenum Press 1981

10. Faris, W.: Self-adjoint operators. Lecture notes in mathematics, Vol. 433. Berlin, Heidelberg, New York: Springer 1975

11. Hirschman, I. I., Widder, D.V.: The convolution transform. Princeton University Press 1955

12. Hörmander, L.: Math. Z. 146, 69-91 (1976)

13. Hunziker, W.: J. Math. Phys. 7, 300-304 (1966)

14. Ikebe, T.: J. Func. Anal. 20, 158-177 (1975)

15. Ikebe, T.: Publ. RIMS Kyoto Univ. 11, 551-558 (1976)

16. Ikebe, T., Isozaki, H.: Publ. RIMS Kyoto Univ. 15, 679-718 (1979)

17. Ikebe, T., Isozaki, H.: Preprint, Kyoto University, 1980

18. Isozaki, H.: Publ. RIMS Kyoto Univ. 13, 589-626 (1977)

19. Jensen, A.: Manuscripta Math. 25, 61-77 (1978)

20. Jensen, A.: Duke Math. J. 47, 57-80 (1980)

21. Jensen, A., Kato, T.: Duke Math. J. 46, 583-611 (1979)

22. Kitada, H.: Proc. Jpn. Acad. Sci. 52, 409-412 (1976)

23 Kitada, H.: J. Math. Soc. Jpn. 29, 665-691 (1977)

24. Kitada, H.: J. Math. Soc. Jpn. 30, 603-632 (1978)

25. Morgan, J., Simon, B.: To be published

26. Mourre, E.: Comm. Math. Phys. 68, 91-94 (1979)

27. Perry, P. A.: Duke Math. J. 47, 187-193 (1980)

28. Reed, M., Simon, B.: Methods of modern mathematical physics. I. Functional analysis. New York: Academic Press 1972

29. Reed, M., Simon, B.: Methods of modern mathematical physics. III. Scattering theory. New York: Academic Press 1979

30. Reed, M., Simon, B.: Methods of modern mathematical physics. IV. Analysis of operators. New York: Academic Press 1978

31. Saitō, Y.: Osaka J. Math. 14, 37-53 (1977)

32. Saito, Y.: Spectral representations for Schrödinger operators with long-range potentials. In: Lecture notes in mathematics, Vol. 727. Berlin, Heidelberg, New York: Springer 1979

33. Simon, B.: Duke Math. J. 46, 119-168 (1979)

34. Strichartz, R.: J. Math. Mech. 16, 1031-1060 (1967)

Communicated by B. Simon

Received November 4, 1980; in revised form April 13, 1981 
